\title{
ResearchOnline@JCU
}

This is the author-created version of the following work:

Romero-Rivas, Carlos, Thorley, Craig, Skelton, Katie, and Costa, Albert (2019) Foreign accents reduce false recognition rates in the DRM paradigm. Journal of Cognitive Psychology, 31 (5-6) pp. 507-521.

Access to this file is available from:

https://researchonline.jcu.edu.au/58768/

(C) 2019 Informa UK Limited, trading as Taylor \& Francis Group.

Please refer to the original source for the final version of this work:

https://doi.org/10.1080/20445911.2019.1634576 


\section{Foreign accents reduce false recognition rates in the DRM paradigm}

Carlos Romero-Rivas $^{\text {a, b }}$, Craig Thorley ${ }^{c}$, Katie Skelton ${ }^{d}$, \& Albert Costa (†) ${ }^{\text {b, e }}$

${ }^{a}$ Department of Evolutive and Educational Psychology, Universidad Autónoma de Madrid, Spain

${ }^{b}$ Center for Brain and Cognition, Universitat Pompeu Fabra, Barcelona, Spain

${ }^{c}$ Department of Psychology, James Cook University, Australia

${ }^{d}$ Department of Psychological Sciences, University of Liverpool, UK

eInstitució Catalana de Recerca I Estudis Avançats (ICREA), Barcelona, Spain

Corresponding author: Carlos Romero-Rivas, Department of Evolutive and Educational Psychology, Universidad Autónoma de Madrid. c/Francisco Tomás y Valiente, 3, 28049 Madrid (Spain). Email: carlos.romeror@uam.es

† Deceased 10 December 2018 


\section{Highlights}

- The DRM paradigm was used to explore whether semantic activation is reduced when processing foreign-accented speech.

- Across two experiments, false recognition of non-studied semantic associates was lower when word lists were presented in a foreign accent, compared to native speech.

- The above results suggest semantic activation may be reduced when processing foreign-accented speech.

- Additionally, it was found that when the foreign speaker had a mild accent, correct recall of studied words was uninfluenced. If the foreign speaker had a strong accent, however, correct recall of studied words was reduced. 


\section{Abstract}

More cognitive resources are required to comprehend foreign-accented than native speech. Focusing these cognitive resources on resolving the acoustic mismatch between the foreign-accented input and listeners' stored representations of spoken words can affect other cognitive processes. Across two studies, we explored whether processing foreign-accented speech reduces the activation of semantic information. This was achieved using the DRM paradigm, in which participants study word lists and typically falsely remember non-studied words (i.e., critical lures) semantically associated with the studied words. In two experiments, participants were presented with word lists spoken both by a native and a foreign-accented speaker. In both experiments we observed lower false recognition rates for the critical lures associated with word lists presented in a foreign accent, compared to native speech. In addition, participants freely recalled more studied words when they had been presented in a native, compared to a foreign, accent, although this difference only emerged in Experiment 2, where the foreign speaker had a very strong accent. These observations suggest that processing foreign-accented speech modulates the activation of semantic information.

Keywords: foreign-accented speech; semantic processing; DRM paradigm; recognition; false memory 


\section{Introduction}

When speech is acoustically degraded, or when speech is produced in non-canonical ways, listeners must recruit additional cognitive resources to make sense of it (Rönnberg et al., 2013; Van Engen \& Peelle, 2014). Crucially, since listeners’ cognitive resources are limited, focusing these resources on resolving acoustic challenges can affect other cognitive processes (Van Engen \& Peelle, 2014). Of most relevance here is the observation that degraded speech reduces the availability of semantic information during on-line comprehension; for instance, when sentence context is low-pass filtered, semantic information is more difficult to encode and therefore presented words are less effectively activated (Aydelott \& Bates, 2004). While previous studies have focused on the effects of acoustic distortions created in the laboratory on speech comprehension, little attention has been paid to the impact of more natural sources of effortful listening, such as foreignaccented speech. It is important to note that extrinsic sources (such as noise or degraded speech) affect the perceptual integrity of the acoustic signal, whereas accent variations affect specific phonemic and suprasegmental features. These differences lead to distinctive brain activation patterns when processing accented speech and other distortions (Adank, Nuttall, Banks, \& Kennedy-Higgins, 2015). Therefore, it is not clear whether extrinsic sources of distorted speech and accent variations would have a similar impact on the same cognitive processes. The purpose of the present study is to explore whether foreign-accented speech reduces the activation of semantic information.

Foreign-accented speech, relative to native speech, is usually less intelligible (Munro \& Derwing, 1995a) and requires more processing time (Munro \& Derwing, 1995b) than native speech. Importantly, the additional cognitive resources needed for lexical processing when listening to foreign-accented speech (Van Engen \& Peelle, 2014) may alter semantic processing. For example, anticipation processes during sentence comprehension are modulated by the speaker's accent: listeners are more likely to preactivate words semantically related to sentences' best completions during native speech comprehension than during foreign-accented speech comprehension (e.g., listeners are more likely to pre-activate the word 'chest' whilst listening to the sentence 'In the pirates' map there was an X showing the location of the...' when uttered by a native speaker, despite expecting the word 'treasure' to appear; Romero-Rivas, Martin, \& Costa, 2016). Also, listeners are slower and less accurate when assessing the veracity of statements presented in a foreign accent (Adank, Evans, Stuart-Smith, \& Scott, 2009). These 
observations suggest that foreign-accented speech makes the encoding of semantic information more difficult during on-line comprehension (Adank et al., 2009; RomeroRivas et al., 2016).

In order to test whether foreign-accented speech reduces the activation of semantic information, we used the DRM paradigm (Deese, 1959; Roediger \& McDermott, 1995). This paradigm is a popular method of inducing false memories and can be used to assess semantic processing. A false memory is an illusory recollection of a non-existent episode or a distorted recollection of an actual episode. Critically, false memories in the DRM paradigm occur as a result of the semantic association between studied and non-studied information (e.g., Roediger \& McDermott, 1995; Howe, Wimmer, Gagnon, \& Plumpton, 2009). More specifically, in this paradigm the participants study lists of words (e.g., "nurse", "hospital", and "patient") that all converge on a single non-studied semantic associate known as the critical lure (e.g., “doctor”). When studying these words, semantic activation spreads to the critical lure and it becomes activated, either implicitly or explicitly, in participant's minds (Dewhurst, Bould, Knott, \& Thorley, 2009). If there is sufficient semantic activation spreading towards the critical lure, participants falsely remember the critical lure as being part of the studied list (Roediger \& McDermott, 1995; McDermott, 1996; Roediger, Balota, \& Watson, 2001; Thapar \& McDermott, 2001; McCabe et al., 2004; Thomas \& Sommers, 2005; Castel et al., 2007; Howe et al., 2009). Conversely, if the critical lure does not receive enough semantic activation, false memories are reduced (Roediger, Watson, McDermott, \& Gallo, 2001).

As an alternative explanation for the usual outcomes observed in the DRM paradigm, the Fuzzy Trace Theory (Brainerd \& Reyna, 1990) argues that studied items contain both a verbatim representation (e.g., visual or auditory details) and a gist representation (e.g., the meaning and semantic content of the studied word). Veridical memory for studied items may occur either by the retrieval of the verbatim or gist representations. However, false memory for critical lures should only contain a gist representation (e.g., the meaning of the critical lure, activated when studying the word list; Brainerd \& Reyna, 1998). Both semantic activation and gist representations could be framed as error-inflating processes, and both support the same prediction about false remembering when DRM word lists are presented to participants in foreign-accented speech: if a foreign accent hinders the spread of the activation from studied words to the 
critical lures, or reduces the extraction of gist representations, false memory rates should be reduced.

Across two studies, we presented participants with DRM word lists spoken by both native and foreign-accented speakers (with each speaker reading out different lists, creating a within-subjects factor). After listening to each word list, participants were presented either with a free recall or a math problems task (as in the original DRM study by Roediger \& McDermott, 1995). Finally, after listening to all word lists, participants were asked to complete a recognition test that included studied items, critical lures, and new items. Recognition of studied items would imply that listeners identified these words when listening to the word lists (and that they properly understood native and foreignaccented words). False recognition of critical lures would imply that listeners activated the semantic associative connections from studied items to critical lures when listening to the word lists. New items served as a baseline, to make sure that participants were properly carrying out the task and not making spurious errors or guesses at retrieval. It is important to note that the recall task (relative to the math task) usually enhances later false recognition of critical lures (Roediger \& McDermott, 1995; Roediger et al., 2004; but see also Roediger, McDermott, \& Robinson, 1998). Consequently, the recall task could facilitate subsequent false recognition in both studies, increasing the overall number of false recognition rates, which may give us more opportunities to find differences between accents.

Interestingly, Sumner and Kataoka (2013) already employed the DRM paradigm to explore whether standard and high/low prestige regional accents modulate semantic encoding and retrieval. In their study, General American (GA) listeners heard DRM lists uttered by GA, British English (high prestige regional accent), and New York non-rhotic (low prestige regional accent) speakers. Sumner and Kataoka (2013) observed that whilst veridical recall rates were nearly identical across speakers, false recall rates were higher for word lists uttered by a low prestige regional accent speaker compared to the other two speakers (and there were no differences between the GA and high prestige regional accented speakers). They concluded that decreased attention to the low prestige regional accent increased gist encoding of the word lists, increasing false recall rates.

Although in our study we did not consider the prestige of the foreign accents, this pattern of results led us to consider contrasting hypotheses. On the one hand, if foreign- 
accented speech makes the encoding of semantic information more difficult (Adank et al., 2009; Romero-Rivas et al., 2016), we expected lower false memory rates when the DRM word lists were presented in foreign-accented speech. On the other hand, if listeners reduce their attention towards word lists uttered by the foreign-accented speakers, we expected higher false memory rates for these lists (Sumner \& Kataoka, 2013). Any of these outcomes would support models of speech processing stating that information about the surface form of speech (i.e., accent) can influence word meaning access (e.g., Cai et al., 2017), contrary to some influential models of word recognition in which the input is abstracted away from some surface-level details (e.g., McClelland \& Elman, 1986; Mirman, McClelland, \& Holt, 2006; Norris, 1994).

As a secondary objective, both studies also explored whether foreign-accented speech reduces studied word recall. Previous studies showed that degraded speech makes it less likely that studied words will be recalled (Rabbitt, 1968; Cousins, Dar, Wingfield, \& Miller, 2014), and that words uttered with unfamiliar accents (such as regional variations) are more difficult to recall/recognize than words spoken in familiar/native accents (Clopper, Tamati, \& Pierrehumbert, 2016; Grohe \& Weber, 2018; but see also Cho \& Feldman, 2013, for a study in which foreign variations were better remembered than native ones). Furthermore, even when degraded spoken words are recognizable by listeners, those that are more difficult to process are less likely to be recalled (Cousins et al., 2014). Thus, in the present studies, we examined whether this is also the case when participants listen to foreign-accented speech.

\section{Experiment 1}

\subsection{Method}

\subsubsection{Subjects}

Thirty-one subjects from the Center for Brain and Cognition (Universitat Pompeu Fabra) database participated in this experiment for monetary compensation (5€). All were native Spanish speakers and reported low familiarity with French accented speakers (after the experiment, none of them reported being familiarized with/having regular contact with French accented speakers). Data from five participants were omitted from the analyses for different reasons (i.e., outlier response times, not following procedural instructions). Thus, 26 participants were included in the final analysis (17 women, all right handed, 
mean age $=23.13$, range $=18-31$ years $)$. A power analysis was conducted in $G^{*}$ Power 3.1 (Faul, Erdfelder, Buchner, \& Lang, 2007). It showed that only 23 participants were necessary in an analysis with three two-levels factors in order to achieve a Power of .95 if alpha was set at .05 and we anticipated a medium effect size $(f=.25)$. This effect size was based on previous research using the DRM paradigm to explore differences in false recall rates between standard and regional accents (Sumner \& Kataoka, 2013).

\subsubsection{Materials}

We adapted 48 Spanish DRM word lists from Alonso, Fernández, Díaz, and Beato (2004) (see Supplementary Material A). Each list contained 15 words that were semantically associated with a non-presented word (critical lure), ordered from the word with the strongest semantic association to the critical lure to the word with the weakest association. We chose these lists because their false recognition rates in Alonso et al.'s (2004) study were always above 25\% (26-98\%). Crucially, we replaced some words with their closest semantic associate (using the free association norms in Spanish by Fernández, Diéz, \& Alonso, 2003). This was done because these words appeared as either a studied word or critical lure in another studied list. For instance, the word "canción” (song) is the critical lure for a list of words in Alonso et al.'s (2004) DRM lists, but it also appeared as a studied item for the list that has "guitarra" (guitar) as the critical lure. We also replaced some words that were repeated across word lists as different lexical variations (such as verb forms, plurals...). We split the 48 word lists into three sets of 16 lists, in order to avoid the repetition of semantic fields inside each set (for instance, including the "canción" and "guitarra” word lists in the same set), and to balance for recognition rates in the original study by Alonso et al. (2004). Each list was recorded digitally by both a native Spanish and a French-accented male speaker, using a sound card running at 44.1 $\mathrm{Hz}$ sampling rate with 32 bits resolution. The foreign-accented speaker was presented with native spoken versions of the words before recording each list, in order to minimize possible differences in speech rate and prosody. Each speaker produced the words in two rounds: one time following the list order during the recordings, and another time following a random order. All of the words were then cut from the auditory files, and we chose the better utterances (e.g., proper prosody, no mispronunciations). All chosen words were balanced in terms of intensity $(\mathrm{dB})$, normalizing the maximum amplitude across all experimental words using the software Audacity®. 
Accent strength of the native and foreign-accented speakers was rated by an independent sample of participants who took part in an unrelated experiment $(\mathrm{N}=22)$. These participants were also native Spanish speakers, selected from the Center for Brain and Cognition database. Each participant listened to a random selection of 20 words spoken by the native Spanish speaker and 20 words spoken by the French foreignaccented speaker of Spanish. They had to rate the strength of the accent for each word from 1 (native accent) to 5 (very strong foreign accent). Listeners rated the Spanish native speaker as non-accented (1.05 out of 5). The French speaker of Spanish was rated with a mild accent ( 2.87 out of 5$)(t(21)=14.23, p<.001, d=20.71)$. That is, the French speaker was perceived as having a stronger accent than the native speaker.

Intelligibility of the speakers was rated by another set of participants who did not take part in the DRM experiment $(\mathrm{N}=9)$. Intelligibility ratings were generated for all items. Participants recognized $100 \%$ of the native spoken words and $98 \%$ of the Frenchaccented words, $t(8)=1.54, p=.08, d=1.50$. That is, the native Spanish speaker was slightly more intelligible than the French-accented speaker, although the difference did not reach significance ${ }^{1}$.

After listening to each auditory list, participants had to complete either a recall or math task. Subsequently, after listening to the 16 auditory lists, participants were presented with a surprise recognition test. Importantly, as mentioned in the introduction, the recall task (relative to the math task) usually enhances later false recognition of critical lures (e.g., Roediger \& McDermott, 1995). The recognition test consisted of 128 words that were randomly presented, one at a time, on a computer screen. 48 were taken from the studied lists, 16 were the critical lures, and 64 were unrelated and new items. The 48 studied items were obtained by selecting three items from each of the 16 presented lists (always from the serial positions 1, 7, 10). The 64 new items were obtained by selecting the unrelated lures and items 1, 7, and 10 from non-studied lists (lists from a different set).

\footnotetext{
${ }^{1}$ However, different studies have shown no relationship between intelligibility and comprehensibility for accented speech (e.g., Derwing \& Munro, 1997; Weil, 2003). Thus, "an accented speech sample can be rated as highly intelligible, but difficult to process at the same time” (Floccia, Butler, Goslin, \& Ellis, 2009).
} 


\subsubsection{Design}

The study had a within-subjects design. Each participant listened to one of the three sets of 16 auditory lists (8 lists spoken by the Spanish native speaker, and 8 lists spoken by the French-accented speaker). There were four versions of each set (changing the accent in which the auditory lists were spoken, and whether each list was followed by the recall or math task, crossing both factors in the randomization), creating a total of 12 experiment versions (that is, each set of 16 auditory lists had 4 possible versions). The version participants completed was randomly selected by the presentation software.

Dependent variables for the recognition test were the hit rate for studied words and the percentage of critical lures falsely recognized after each task. We also computed non-parametric signal detection indices of sensitivity (A') and response bias (B', d) (see below for further information). Dependent variables for the recall task were the percentage of studied words correctly recalled, and percentage of critical lures falsely recalled.

\subsubsection{Procedure}

Participants were tested individually at a computer terminal in a sound-attenuated room. They were told that they would be taking part in a study examining foreign-accented speech comprehension and that they would listen to lists of spoken words over headphones. Items were blocked by list at encoding, and presentation of each word list was randomized (i.e., each participant was presented with a different order). After listening to each individual list, participants were presented with a tone and a word written in the middle of the screen. If the word was "Recall”, participants had to pick a sheet of paper from a stack on their right and write down as many words as they could remember from the auditory list they had just listened to. If the word was “Math”, participants had to pick a sheet from a stack on their left and complete as many operations as they could. After one minute, a second tone sounded and participants were told to place the sheet they just completed under the corresponding stack. The inter-stimulus interval was 1 second within lists.

Next, participants were asked to read the self-paced recognition test instructions. They were told they would see one word at a time on the screen and that they would be required to indicate, using the keyboard, whether the item had been studied before or not. 


\subsection{Results}

Prior to all analyses, statistical assumptions were checked and, unless otherwise stated, met. The descriptive statistics associated with Experiment 1 are in Table 1.

\subsubsection{Recognition}

To evaluate the effect of talker information on recognition, results were submitted to logit mixed models, evaluating how often a subject made a "yes" response (identifying an item as old) as a function of the fixed factors item type (studied, critical), the task completed immediately after hearing each list (maths, recall), and the accent of the spoken word lists (native, foreign) ${ }^{2}$. Random effects included intercepts for subjects and items, as well as random by-subject slopes for the effects of item type and accent, and random by-subject interactions between these factors.

The analysis revealed a main effect of $\operatorname{task}(\beta=4.67, S E=1.08,|z|=4.34, p<$ .001), with participants identifying more items as old when the recognition test followed the recall task (compared to the math task), and a two-way interaction between item type and accent, $(\beta=1.76, S E=0.51,|z|=3.48, p<.001)$. Data were then partitioned along item type, with follow-up logit mixed models analysing each type of item. The main effect of accent was not significant in the analysis of studied items $(\beta=0.31, S E=0.40,|z|=$ $0.77, p=.44)$. However, the main effect of accent was significant in the analysis of critical lures $(\beta=4.73, S E=2.37,|z|=1.99, p=.046)$, with participants identifying more critical lures as old when they were associated with word lists studied in the native compared to the foreign accent (see Table 1 and Figure 1).

Additionally, participants also incorrectly recognized $18 \%(S E=.03)$ of the new items (i.e., those that were not presented in the word lists or semantically associated with any of the words on the lists).

\footnotetext{
${ }^{2}$ Logit mixed models were used in the analyses of recognition rates to avoid problems associated with using ANOVAs when analysing categorical data (Jaeger, 2008). R (R Core Team, 2016) scripts from Luthra, Fox, and Blumstein (2018; osf.io/5b7ct) were adapted for our purposes, using the glmer function of the lme4 package (Bates, Maechler, Bolker, \& Walker, 2015). For further details on the models and scripts, please refer to Luthra et al. (2018).
} 


\subsubsection{Signal detection theory}

In addition to examining participants' mean levels of false and studied word recognition, we also calculated non-parametric signal detection indices ${ }^{3}$ of sensitivity and response bias. Sensitivity refers to the ability to discriminate between studied and non-studied items, whereas response bias refers to the likelihood of classing an item as studied. These measures allowed us to examine whether participants falsely recognized more critical lures associated with the native, compared to the foreign-accented, word lists as they had a lower sensitivity towards detecting these lures and/or because they had a more liberal response bias when responding to them (for further information on signal detection theory measures, please see Stanislaw \& Todorov, 1999).

To achieve this, we computed two sets of non-parametric signal detection indices of sensitivity $\left(A^{\prime}\right)$ and response bias $\left(B^{\prime \prime}{ }^{\prime}\right)$. The first set compared hits towards studied words to false recognition of critical lures (henceforth $A$ ' Critical lure and B' ${ }_{d}$ Critical lure), and the second set compared hits towards studied words to false alarms for new, unrelated words (henceforth A' New items and B' 'd New items; see Ost, Blank, Davies, Jones, Lambert, \& Salmon, 2013, for a similar approach). Values of $A^{\prime}$ range from 0 (no discrimination) to 1 (perfect discrimination), and values of $B$ '” ${ }_{d}$ range from - 1 (liberal bias) to +1 (conservative bias). For each measure, we carried out a paired samples t-test comparing performance when lists were presented in the two accents (native, foreign).

Data were not normally distributed, and therefore we used the Wilcoxon Signed Rank test to analyse differences in sensitivity and response bias between accents. For the $A^{\prime}$ Critical lure and $A^{\prime}$ New item measures, participants were equally likely to discriminate between studied and non-studied words, regardless of accent (both $p$ 's > .34). For $B$ ”' ${ }_{d}$ Critical lure, participants were liberal in their responses and were more likely to class words as studied when associated with the native ( $M d=-0.97)$ compared to the foreign-accented speaker ( $M d=-0.90), T=214.50, n=26, z=-2.33, p=.02, r=$ .46. For $B$ '” ${ }_{d}$ New items, participants were conservative in their responses and were equally likely to class these items as studied, irrespective of accent $(p=.22)$. Combined, the findings discussed so far imply that participants falsely recognized less critical lures when they were associated with foreign-accented word lists as they had a less liberal

\footnotetext{
${ }^{3}$ We used non-parametric indices because the recognition data were negatively skewed (see Figure 1).
} 
response bias towards these non-studied words (i.e., they were less likely to class them as studied).

\subsubsection{Recall}

Data were not normally distributed, so we used the Wilcoxon Signed Rank test to examine whether presenting word lists in a native or foreign accent influenced subsequent critical lure false recall and, separately, studied word recall. Accent had no effect on either measure (both $p$ 's $>.88$ ).

\subsection{Discussion}

In this experiment, we used the DRM paradigm to explore whether semantic activation is reduced when processing foreign-accented speech. Furthermore, in an exploratory analysis we investigated whether foreign-accented speech reduces the veridical recall of studied words, as is the case during degraded speech comprehension (Rabbitt, 1968; Cousins et al., 2014). Our main findings were that:

- First, word lists spoken in a foreign accent elicited lower critical lure false recognition rates than those presented in a native accent

- Second, participants had a less liberal response bias towards critical lures when they were associated with word lists spoken in foreign-accented (i.e., they were less likely to class them as studied)

- Finally, studied word recall was not influenced by the accent of the spoken word lists

The fact that foreign-accented speech elicits lower false recognition rates for the critical lures than native speech supports previous findings suggesting that foreignaccented speech hinders semantic information processing during speech comprehension (Adank et al., 2009; Romero-Rivas et al., 2016). However, this observation should be treated with caution, because this outcome seems to be mostly explained by differences in response biases towards native and foreign-accented speech, rather than by sensitivity when discriminating between studied items and critical lures. It is important to note that the differences in response biases observed when items had been presented in native and foreign-accented speech were only significant when taking into account studied items and critical lures ( $B$ ', ${ }_{d}$ Critical lure), and not when taking into account studied and new, unrelated, items ( $B$ '” New items); consequently, the effect seems to be mainly driven by 
critical lures, rather than by studied items. Thus, participants showed a clear tendency to respond old to critical lures associated with word lists studied in native speech (that is, their criterion for classing a critical lure as studied was liberal and they readily did so); however, their criterion for responding old to critical lures associated with word lists studied in foreign-accented speech was higher (that is, their criterion for classing a critical lure as studied was stricter and they were less likely to do so).

A tentative explanation for these results is that native and foreign accents lead listeners to pay different levels of attention to the semantic (gist representations) and phonological features (verbatim representations) of words. Listeners have to deal with an effortful listening condition when presented with foreign-accented speech, because of the mismatch between incoming speech and listeners' representations. Accordingly, listeners' resources are focused on phonological and lexical information, in order to adapt to the variations introduced by the accented speech (e.g., Witteman et al., 2015). Therefore, listeners might focus more attention on verbatim representations during the study of word lists spoken by the foreign-accented speaker as compared to word lists spoken by the native speaker. Later, they may adopt a less liberal response bias towards words associated with foreign-accented speech during the recognition test, in such a way that the words matching phonological and lexical representations that were recently activated (either during the study or the free recall of the word lists) would be more easily recognized as studied items. Regarding native speech, listeners would pay attention to both verbatim and gist representations during the study phase, adopting a more liberal response bias during the recognition test, in such a way that words matching recently activated semantic representations would also be easily recognized as studied items. This suggestion is consistent with previous results showing that listeners retrieve the semantic properties of words in a more effective manner when listening to native as compared to foreign-accented speech (Adank et al., 2009; Romero-Rivas et al., 2016) and degraded speech (Aydelott \& Bates, 2004; Aydelott et al., 2006).

This explanation may also help us to interpret the differences between our results and the observations made by Sumner and Kataoka (2013), who found higher false recall rates for low prestige regional accented speakers compared to standard and high prestige regional accented speakers. Whilst in our study participants may be paying more attention to acoustic-phonetic details (verbatim representations) of foreign-accented words (because foreign-accented speech is particularly difficult to process), participants in 
Sumner and Kataoka's (2013) study may have not paid as much attention to the word lists uttered by the low prestige regional accented speaker. Consequently, participants in Sumner and Kataoka's (2013) study may have depended on gist representations to a higher extent when listening to the low prestige regional accented speaker compared to the standard and high prestige regional accented speakers, resulting in inflated levels of false remembering (as gist encoding is more frequently observed in decreased attention conditions; Otgaar, Peters, \& Howe, 2012).

As for the recall task, and as mentioned in the introduction, previous studies have shown that degraded speech negatively affects the recall of word lists (Rabbitt, 1968), that distorted words that are particularly difficult to process are less likely to be later recalled (Cousins et al., 2014), and that unfamiliar regional accents lead to worst recall/recognition of studied words than familiar/native accents (Clopper et al., 2016; Grohe \& Weber, 2018; but see Cho \& Feldman, 2013). Nevertheless, when exploring whether a mild foreign accent reduced the veridical recall of word lists, we found this was not the case. It may be the case that a mild foreign accent does not affect retrieval processes during the recall task, but that stronger accents could actually modulate these same processes (which would be in line with previous findings indicating that degraded words that are particularly difficult to process are less likely to be recalled than other, easier to process, degraded words; Cousins et al., 2014).

To explore this possibility, and to test the generalizability of our observation that critical lure false recognition decreases when the lures are associated with word lists presented in a foreign accent, we carried out a second experiment using new DRM lists presented in a stronger foreign accent.

\section{Experiment 2}

In this experiment, we presented native speakers of English with DRM word lists spoken both by a native speaker and a Spanish foreign-accented speaker of English. This decision was based on the fact that English and Spanish belong to different language families (English is in the Germanic family, and Spanish in the Latin family), and thus have many differences in terms of pronunciation and phonological patterns. Critically, this may increase the perception of the Spanish foreign-accented speaker of English as a speaker with a strong foreign accent. 
As in the previous experiment, participants were presented with either a free recall or a math task after listening to each word list, and with the recognition test after listening to all word lists. We hypothesized that the stronger foreign accent in Experiment 2 would make the encoding of semantic information more difficult than the native accent (Adank et al., 2009; Romero-Rivas et al., 2016), and therefore we would replicate our previous finding showing that foreign-accented speech induces lower levels of false recognition for the DRM word lists than native speech. Furthermore, if a strong foreign accent can modulate immediate recall processes (as it is the case for degraded words that are particularly difficult to process; Cousins et al., 2014), we would expect that the strong foreign accent would also reduce studied word recall.

\subsection{Method}

\subsubsection{Subjects}

Thirty-six University of Liverpool undergraduates participated in this study for course credit or monetary compensation (£10). All were native English speakers and reported low familiarity with Spanish accented speakers (after the experiment, none of them reported being familiarized with/having regular contact with Spanish accented speakers). Data from eight participants were omitted from the analyses for several reasons (i.e., improbably fast response times, not following procedural instructions). Thus, 28 participants were included in the final analyses (24 women, 27 right-handed, mean age $=$ 21.35 years, range $=18-33$ years $)$.

\subsubsection{Materials}

Given that translated lists usually elicit weaker associative relationships between studied items and critical lures (Graves \& Altarriba, 2014), we used different DRM lists for this experiment. We took the 16 DRM word lists that elicited the highest false recognition rates in Stadler et al.’s (1999) study (see Supplementary Material B). Each list consisted of 15 words that were strongly associated with a non-presented word (critical lure), ordered from the word with the strongest semantic association to the critical lure to the word with the weakest association. Each list was digitally recorded in English by a female with a native English accent and a female with a foreign Spanish accent, using a sound card running at a $44.1 \mathrm{~Hz}$ sampling rate with 32 bits resolution. Recording and editing of the audio files followed the same procedures as in Experiment 1. We used items from an 
additional set of 16 word lists, developed by Roediger and McDermott (1995) and McDermott (1996), as non-studied fillers during the recognition test.

An independent sample of participants, also University of Liverpool undergraduates, rated the experimental stimuli in terms of intelligibility and accent strength $(\mathrm{N}=9)$. Regarding intelligibility, listeners were presented with all items and recognized $94 \%$ of the native spoken words $(S D=0.02)$, and $90 \%$ of the foreign-accented words $(S D=0.04)(t(8)=1.87, p=.05, d=1.26)$. That is, the native English speaker was marginally, but not significantly, more intelligible than the Spanish-accented speaker. Regarding accent strength ratings, listeners always rated the native English speaker as non-accented (1 out of 5). The Spanish-accented speaker was rated with a stronger accent (4.11 out of 5$)(t(8)=10.90, p<.001, d=6.30)$. That is, the Spanish-accented speaker was perceived as having quite a strong foreign accent, while the English speaker was perceived as a native. We also carried out a Welch's t-test (due to the unequal sample sizes for participants completing the ratings in Experiments 1 and 2; see Delacre, Lakens, \& Leys, 2017) comparing the strength of the foreign accents across both our experiments, and found that the foreign-accented speaker in Experiment 2 was perceived as having a stronger accent than the foreign-accented speaker in Experiment $1, t(11.81)=4.31$, $p<.001, d=2.51$.

As in the previous experiment, participants had to complete either a recall or a math task after each auditory list. The recognition test was similar to the one used in the previous experiment, with the only difference being that the 64 new items (items not presented and not related to the studied items) were obtained by selecting the unrelated lures and items 1,7 , and 10 from the lists that elicited lower false recognition rates in Stadler et al.’s (1999) study (that is, the lists that we did not include as experimental lists).

\subsubsection{Design}

Each participant listened to the 16 auditory lists (8 lists spoken by the native Englishaccented speaker, and 8 lists spoken by the foreign Spanish-accented speaker). There were 4 experimental sets (changing the accent in which the auditory lists were spoken, and whether each list was followed by the recall or math task, crossing both factors in the randomization). The version participants listened to was randomly selected by the presentation software. Independent and dependent variables were the same as in the previous experiment. 


\subsubsection{Procedure}

Participants were tested in groups of two to four in a sound-attenuated room, with each sat in a separate, partitioned, computer booth. The rest of the procedure was identical to Experiment 1's.

\subsection{Results}

Prior to all analyses, statistical assumptions were checked and, unless otherwise stated, met. The descriptive statistics associated with Experiment 2 are in Table 2.

\subsubsection{Recognition}

As in Experiment 1, results were submitted to logit mixed models, evaluating how often a subject made a "yes" response (identifying an item as old) as a function of the fixed factors item type (studied, critical), task completed immediately after hearing each list (maths, recall), and accent of the spoken word lists (native, foreign). Also as in Experiment 1, random effects included intercepts for subjects and items, as well as random by-subject slopes for the effects of item type and accent, and random by-subject interactions between these factors.

The analysis revealed a main effect of $\operatorname{task}(\beta=6.92, S E=1.25,|z|=5.53, p<$ .001 ), with participants identifying more items as old when the recognition test followed the recall task (compared to the math task), and a two-way interaction between item type and accent, $(\beta=2.14, S E=0.52,|z|=4.11, p<.001)$. Data were then partitioned along item type, with follow-up logit mixed models analysing each type of item. The main effect of accent was not significant in the analysis of studied items $(\beta=1.14, S E=0.77,|z|=$ $1.49, p=.14)$. However, the main effect of accent was significant in the analysis of critical lures $(\beta=2.52, S E=0.001,|z|=2083, p<.001)$, with participants identifying more critical lures as old when they were associated with word lists studied in the native compared to the foreign accent (see Table 2).

Additionally, participants falsely recognized $13 \%(S E=.03)$ of the new items (i.e., those that were not presented in the word lists or semantically associated with any of the words on the lists). 


\subsubsection{Signal detection theory}

Following the same logic as in Experiment 1, we computed two sets of non-parametric signal detection indices of sensitivity $\left(A^{\prime}\right)$ and response bias $\left(B^{\prime \prime}{ }_{d}\right)$. For each measure, we compared performance when words were presented in the two accents (native, foreign).

As in Experiment 1, data were not normally distributed, and therefore we used the Wilcoxon Signed Rank test to analyse differences in sensitivity and response bias between accents. For A' Critical lure and A' New items, participants were equally likely to discriminate between studied and non-studied words, irrespective of accent (both $p$ 's $>$.07). As in Experiment 1, the $B$ "' ${ }_{d}$ Critical lure measure revealed that participants were quite liberal in their responses and were more likely to class words as studied when associated with the native $(M d=-0.92)$ compared to the foreign-accented speaker $(M d=$ -0.72), $T=202, n=28, z=-1.95, p<.05, r=.37$. The $B$ ' ${ }_{d}$ New items measure, however, demonstrated participants had a more conservative leaning response bias when making decisions involving new items, and were slightly less conservative in the native accent conditions, but the effect of accent was not significant $(p=.08)$. Combined, these findings again suggest that participants falsely recognized less critical lures when they were associated with word lists spoken by the foreign-accented speaker, as they had a less liberal response bias towards these non-studied words.

\subsubsection{Recall}

A paired-sample t-tests was used to examine whether presenting word lists in a native or foreign accent influenced studied word recall, as data were normally distributed. More words were correctly recalled when they had been presented in a native accent, $t(27)=$ 3.04, $p=.005, d=0.55$. However, data for critical lure false recall was not normally distributed, and thus we used the Wilcoxon Signed Rank test to explore whether the speakers' accent modulated false recall. We found that that was not the case $(p=.75)$.

\subsection{Discussion}

In Experiment 2, we explored whether a strong foreign accent affects the activation of semantic information and the immediate veridical recall of word lists in the DRM paradigm. To sum up, our main findings were that: 
- First, DRM word lists spoken in a strong foreign accent elicited lower levels of critical lure false recognition than DRM word lists produced by a native speaker.

- Second, participants had a less liberal response bias when deciding whether critical lures associated with the foreign-accented word lists had been studied or not (i.e., they were less likely to class them as studied).

- Finally, studied word recall was lower when word lists had been spoken in a strong foreign accent.

We therefore replicated and extended the main result from the previous study: that foreign-accented speech (as compared to native speech) reduces false recognition rates for the critical lures. Again, this outcome is consistent with the previous literature showing that foreign-accented speech makes the processing of semantic information more difficult during speech comprehension (e.g., Adank et al., 2009; Romero-Rivas et al., 2016). Importantly, and as we also found in the previous experiment, this finding seems to be driven by response bias differences towards critical lures associated with native and foreign-accented word lists. More specifically, participants were more likely to class critical lures associated with the word lists uttered by the native speaker as having been studied (with a medium sized, albeit marginally non-significant, effect observed).

Regarding the recall task, the observation that participants recalled more words spoken by the native than by the strong foreign-accented speaker is congruent with previous studies showing that degraded speech and regional accents affect the recall of word lists (Rabbitt, 1968; Cousins et al., 2014; Clopper et al., 2016; Grohe \& Weber, 2018). This result, however, contrasts with the previous experiment, where no differences were observed between native and mild foreign-accented speech in the immediate recall of studied items. A tentative explanation for the divergence between the two studies is that foreign accents can modulate immediate recall processes, but only when the foreignaccented speaker has a strong accent. That explanation would be consistent with previous results showing that degraded words which are especially difficult to process are the ones that are less likely to be recalled (compared to degraded words which are processed more easily; Cousins et al., 2014). Also, it would be in accordance with the aforementioned explanation for the false recognition results: participants in Experiment 2 might have needed to allocate more attention to the phonological features (verbatim representations) of the strong foreign-accented words, because of the large differences between English and Spanish (Witteman et al., 2015). Consequently, participants would have less 
resources available to process semantic information (gist representations) when listening to the strong foreign-accented (as opposed to the native) speaker (Witteman et al., 2015), leading to a lower rate of free recall for studied words.

Nevertheless, the results from our recall task contrast with those of Cho and Feldman (2013). They found that recall of spoken unrelated words was superior when the words had been uttered in an unfamiliar accent, relative to native speech. Differences between Cho and Feldman's (2013) materials (listening to or repeating/imitating lists of unrelated words) and ours (listening to lists of semantic associates) may have caused these contrasting results. Semantically associated words are more memorable than unrelated words, as participants focus on the semantic relationships during encoding and use this to assist their recall (Hyde \& Jenkins, 1973). Thus, if participants in our experiment were less able to focus on semantic information when listening to word lists uttered by the foreign-accented speaker, they would be less likely to have this advantage (and would recall less studied words from those lists).

\section{General Discussion}

Results from two experiments using the DRM paradigm revealed that foreign-accented speech elicited lower false recognition rates for the non-studied critical lures than native speech. This finding is consistent with previous studies showing that foreign accents make the processing of semantic information more difficult (Adank et al., 2009; RomeroRivas et al., 2016) and suggests that during foreign-accented speech comprehension, activation of semantic neighbours is more difficult. Importantly, differences between native and foreign-accented speech seemed to be mostly explained by a less liberal response bias towards critical lures associated with the latter. This indicates that listeners' resources might be focused on processing phonological information, and not semantic information, during foreign-accented speech comprehension (Witteman et al., 2015), facilitating the recognition of words matching recently activated phonological representations (i.e., studied words), but possibly lowering the false recognition of semantically related words (i.e. non-studied critical lures). Future studies on this topic may directly test this explanation by using word lists that converge on both phonological and semantic critical lures (e.g., see Luthra et al., 2018).

An alternative explanation for our false memory findings is that the distinctiveness of the foreign-accented speech meant participants processed this information more 
carefully and this reduced false recognition rates in our two experiments (see Schacter \& Wiseman, 2006, for an overview of how distinctiveness impacts upon false remembering). Distinctiveness, however, tends to have opposing effects on false and correct recognition (i.e., a mirror effect pattern; Glanzer \& Adams, 1990). Distinctiveness typically reduces false recognition but improves correct recognition (e.g., Dodson \& Schacter, 2001; Gunter, Bodner, \& Azad, 2007). In our studies, we found that correct recognition did not vary as a function of accent (and, additionally, correct recall was reduced in Experiment 2 when lists had been presented in a foreign-accent). We are therefore confident that distinctiveness effects did not influence our findings.

Furthermore, the observation that listeners freely recalled more words studied in a native accent, compared to a foreign accent, in Experiment 2 (where the foreign speaker had a very strong accent) is congruent with previous findings showing that only those words that are particularly difficult to process (compared to degraded words that are easier to process) are less likely to be recalled (Cousins et al., 2014). Thus, the contrast observed between the results of the first and second experiment (i.e., in the first experiment we did not observe differences between native and foreign-accented speech in the veridical recall of studied items, but in the second experiment we did) might be explained by differences in the strength of the accent of the foreign-accented speakers. Listeners usually need more time to adapt to strong, compared to mild, foreign-accented speech (Witteman et al., 2013, 2015), and they take more time to recognize words in an identity priming task when the strength of the foreign accent is increased (Porretta et al., 2016). Consequently, increasing the strength of the foreign accent in Experiment 2 might have caused listeners to focus their attentional resources on phonological information to a higher extent than during the first experiment (where the strength of the foreign accent was milder; Witteman et al., 2015), leading them to achieve a lower rate of free recall for words studied with the strong foreign accent.

However, an alternative explanation is that the contrast between the two studies might have been caused by inequalities between the DRM lists the participants heard in both experiments. Preparation of the DRM word lists was somewhat similar in Alonso et al. (2004) and Stadler et al. (1999) studies, and false recognition rates were quite similar for the lists extracted from the two studies. Nevertheless, false recall rates for critical lures and free recall rates for studied items were slightly higher in Stadler et al.'s (1999) study, compared to Alonso et al.'s (2004) study. Therefore, the contrast between the two studies 
might be explained by different lexical activations during the recall task. Additionally, Experiments 1 and 2 also differed in the language (Spanish vs. English) and the specific type of foreign accents (French vs. Spanish) used. We acknowledge that these issues are a potential limitation of this study, and that future research should explore whether differences in the strength of the foreign accent modulate the activation of semantic information and recall processes when using the same language and the same DRM word lists.

A somewhat unexpected observation is that we found differences between native and foreign-accented speech in the false recognition of critical lures, but not in the false recall of critical lures. Although listeners in both experiments falsely recalled a slightly higher proportion of critical lures for native, compared to foreign-accented, word lists, these differences were not significant. Previous studies have shown that aging effects on false memories, for instance, are observable both in false recall and recognition (e.g., Norman \& Schacter, 1997) and, consequently, we expected that accent effects in relation to false memories would also be observable when using both measures. A possible explanation for this result is that the lower levels of false recall, relative to false recognition, meant that significant differences between accents were more difficult to detect (Grohe \& Weber, 2018). Alternatively, the differences observed between true and false recall/recognition may be explained by the distinct processes triggered by each task: whilst recall involves a generation phase (e.g., Kintsch, 1970), recognition is based on familiarity and recollection (e.g., James, 1890). Consequently, differences between accents in Experiment 2 for veridical recall may be influenced by how easy it is to generate the studied items, whereas the lower false recognition rates for foreign-accented compared to native speech in both experiments might be modulated by familiarity (as suggested by differences in response biases). However, this issue ought to be examined in future research.

\subsection{Conclusions}

To conclude, we showed that foreign-accented speech reduces false recognition rates in the DRM paradigm. This effect seemed to be mainly explained by a more liberal response bias towards native, relative to foreign-accented speech. Also, we observed that a strong foreign accent reduced the immediate recall of studied items. As an explanation for these results, it could be argued that listeners pay more attention to phonological (i.e., 
less attention to semantic) information when listening to foreign-accented as compared to native speech, causing them to rely on recently activated phonological representations to recognize an item as studied. Our results would be congruent with models of speech processing suggesting that information about the surface form of the speech signal (such as the speaker’s accent) modulates semantic processing (e.g., Cai et al., 2017).

\section{Acknowledgements}

In loving memory of Albert Costa. We thank Xavier Mayoral and Silvia Blanch for their technical support. CRR was supported by a MINECO predoctoral grant for predoctoral researchers from the Spanish government (BES-2012-056668). AC was supported by two grants from the Spanish Government (PSI2011-23033, and PSI2014-52181-P), a grant from the Catalan Government (AGAUR SGR 268), and a grant from the European Research Council under the European Community's Seventh Framework Programme (FP7/2007-2013 Cooperation grant agreement nº 613465 - AThEME). 


\section{References}

Adank, P., Evans, B. G., Stuart-Smith, J., \& Scott, S. K. (2009). Comprehension of familiar and unfamiliar native accents under adverse listening conditions. Journal of Experimental Psychology: Human Perception and Performance, 35(2), 520.

Adank, P., Nuttall, H. E., Banks, B., \& Kennedy-Higgins, D. (2015). Neural bases of accented speech perception. Frontiers in Human Neuroscience, 9(558).

Alonso, M. A., Fernández, A., Díez, E., \& Beato, M. S. (2004). Índices para la producción de falso recuerdo y falso reconocimiento para 55 listas de palabras en castellano. Psicothema, 16, 357-362.

Aydelott, J., \& Bates, E. (2004). Effects of acoustic distortion and semantic context on lexical access. Language and Cognitive Processes, 19(1), 29-56.

Aydelott, J., Dick, F., \& Mills, D. L. (2006). Effects of acoustic distortion and semantic context on event-related potentials to spoken words. Psychophysiology, 43(5), 454-464.

Bates, D., Maechler, M., Bolker, B, \& Walker, S. (2015). Fitting linear mixed-effects models using lme4. Journal of Statistical Software, 67(1), 1-48.

Brainerd, C. J., \& Reyna, V. F. (1990). Gist is the grist: Fuzzytrace theory and the new intuitionism. Special issue: Limited resource models of cognitive development. Developmental Review, 10(1), 3-47.

Brainerd, C. J., \& Reyna, V. F. (1998). When things that were never experienced are easier to "remember"' than things that were. Psychological Science, 9(6), 484-489.

Cai, Z. G., Gilbert, R. A., Davis, M. H., Gaskell, M. G., Farrar, L., Adler, S., \& Rodd, J. M. (2017). Accent modulates access to word meaning: Evidence for a speaker-model account of spoken word recognition. Cognitive Psychology, 98, 73-101.

Castel, A. D., McCabe, D. P., Roediger, H. L. III, \& Heitman, J. L. (2007). The dark side of expertise domain-specific memory errors. Psychological Science, 18(1), 3-5.

Cho, K. W., \& Feldman, L. B. (2013). Production and accent affect memory. The Mental Lexicon, 8(3), 295-319. 
Clopper, C. G., Tamati, T. N., \& Pierrehumbert, J. B. (2016). Variation in the strength of lexical encoding across dialects. Journal of Phonetics, 58, 87-103.

Cousins, K. A. Q., Dar, H., Wingfield, A., \& Miller, P. (2014). Acoustic masking disrupts time-dependent mechanisms of memory encoding in word-list recall. Memory \& Cognition, 42, 622-638.

Deese, J. (1959). On the prediction of occurrence of particular verbal intrusions in immediate recall. Journal of experimental psychology, 58(1), 17-22.

Delacre, M., Lakens, D., \& Leys, C. (2017). Why psychologists should by default use Welch's t-test instead of Student's t-test. International Review of Social Psychology, 30, 92-101.

Derwing, T. M., \& Munro, M. J. (1997). Accent, intelligibility and comprehensibility. Studies in Second Language Acquisition, 19, 1-16.

Dewhurst, S. A., Bould, E., Knott, L. M., \& Thorley, C. (2009). The roles of encoding and retrieval processes in associative and categorical memory illusions. Journal of Memory and Language, 60, 154-164.

Dodson, C. A., \& Schacter, D. L. (2001). "If I had said it I would have remembered it”: Reducing false memories with a distinctiveness heuristic. Psychonomic Bulletin \& Review, 8 (1), 155-161.

Faul, F., Erdfelder, E., Lang, A-G., \& Buchner, A. (2007). G*Power 3: A flexible statistical power analysis program for the social, behavioural, and biomedical sciences. Behavior Research Methods, 39, 175-191.

Fernández, A., Díez, E., \& Alonso, M. A. (2003). Normas de asociación libre en castellano. http://inico.usal.es/usuarios/gimc/normas/index_nal.asp

Floccia, C., Butler, J., Goslin, J., \& Ellis, I. (2009). Regional and foreign accent processing in English: can listeners adapt? Journal of Psycholinguistic Research, 38, 379412.

Glanzer, M., \& Adams, J. K. (1990). The mirror effect in recognition memory: Data and theory. Journal of Experimental Psychology: Learning, Memory, and Cognition, 16(1), 5-16. 
Graves, D. F., \& Altarriba, J. (2014). False memories in bilingual speakers. In Foundations of bilingual memory (pp. 205-221). New York, NY: Springer.

Grohe, A. K., \& Weber, A. (2018). Memory advantage for produced words and familiar native accents. Journal of Cognitive Psychology, 30, 570-587.

Gunter, R. W., Bodner, G. E., \& Azad, T. (2007). Generation and mnemonic encoding induce a mirror effect in the DRM paradigm. Memory \& Cognition, 35(5), 1083-1092.

Howe, M. L., Wimmer, M. C., Gagnon, N., \& Plumpton, S. (2009). An associativeactivation theory of children's and adults’ memory illusions. Journal of Memory and Language, 60(2), 229-251.

Hyde, T. S., \& Jenkins, J. J. (1973). Recall for words as a function of semantic, graphic, and syntactic orienting tasks. Journal of Verbal Learning and Verbal Behavior, 12(5), $471-480$

Jaeger, T. F. (2008). Categorical data analysis: Away from ANOVAs (transformation or not) and towards logit mixed models. Journal of Memory and Language, 59(4), 434-446.

James, W. (1890). The principles of psychology. New York, NY: Holt.

Kintsch, W. (1970). Models for free recall and recognition. In D. A. Norman (Ed.), Models of human memory (pp. 331-370). New York, NY: Academic Press.

Luthra, S., Fox, N. P., \& Blumstein, S. E. (2018). Speaker information affects false recognition of unstudied lexical-semantic associates. Attention, Perception, \& Psychophysics, 80, 894-912.

McCabe, D. P., Presmanes, A. G., Robertson, C. L., \& Smith, A. D. (2004). Item-specific processing reduces false memories. Psychonomic Bulletin \& Review, 11(6), 1074-1079.

McClelland, J. L., \& Elman, J. L. (1986). The TRACE model of speech perception. Cognitive Psychology, 18, 1-86.

McDermott, K. B. (1996). The persistence of false memories in list recall. Journal of Memory and Language, 35(2), 212-230. 
Mirman, D., McClelland, J. L., \& Holt, L. L. (2006). An interactive Hebbian account of lexically guided tuning of speech perception. Psychonomic Bulletin \& Review, 13, 958965.

Munro, M. J., \& Derwing, T. M. (1995a). Foreign accent, comprehensibility, and intelligibility in the speech of second language learners. Language learning, 45(1), 7397.

Munro, M. J., \& Derwing, T. M. (1995b). Processing time, accent, and comprehensibility in the perception of native and foreign-accented speech. Language and speech, 38(3), 289-306.

Norman, K. A., \& Schacter, D. L. (1997). False recognition in younger and older adults: Exploring the characteristics of illusory memories. Memory \& Cognition, 25, 838-848.

Norris, D. (1994). Shortlist: A connectionist model of continuous speech recognition. Cognition, 52(3), 189-234.

Ost, J., Blank, H., Davies, J., Jones, G., Lambert, K., \& Salmon, K. (2013). False memory $\neq$ false memory: DRM errors are unrelated to the misinformation effect. PLOS ONE, 8, e57939.

Otgaar, H., Peters, M., \& Howe, M. L. (2012). Dividing attention lowers children's, but increases adults' false memories. Journal of Experimental Psychology: Learning, Memory, and Cognition, 38, 204-210.

Porretta, V., Tucker, B. V., \& Järvikivi, J. (2016). The influence of gradient foreign accentedness and listener experience on word recognition. Journal of Phonetics, 58, 1-21.

Rabbitt, P. M. A. (1968). Channel capacity, intelligibility and immediate memory. Quarterly Journal of Experimental Psychology, 20, 241-248.

R Core Team. (2016). R: A language and environment for statistical computing [Computer software]. Vienna, Austria: R Foundation for Statistical Computing. Retrieved from http://www.R-project.org/

Roediger, H. L., III, Balota, D. A., \& Watson, J. M. (2001). Spreading activation and arousal of false memories. In H. L. RoedigerIII, J. S. Nairne, I. Neath, \& A. M. Surprenant 
(Eds.), Science conference series. The nature of remembering: Essays in honor of Robert G. Crowder (pp. 95-115). Washington, DC: American Psychological

Association.Roediger, H. L. III, \& McDermott, K. B. (1995). Creating false memories: Remembering words not presented in lists. Journal of experimental psychology: Learning, Memory, and Cognition, 21(4), 803-814.

Roediger, H. L. III, McDermott, K. B., Pisoni, D. B., \& Gallo, D. A. (2004). Illusory recollection of voices. Memory, 12, 586-602.

Roediger, H. L., III, McDermott, K. B., \& Robinson, K. J. (1998). The role of associative processes in creating false memories. In M. A. Conway, S. E. Gathercole, \& C. Cornoldi (Eds.), Theories of memory II (pp. 187-245). Hove: Psychology Press.

Roediger, H. L. III, Watson, J. M., McDermott, K. B., \& Gallo, D. A. (2001). Factors that determine false recall: A multiple regression analysis. Psychonomic Bulletin \& Review, 8(3), 385-407.

Romero-Rivas, C., Martin, C. D., \& Costa, A. (2016). Foreign-accented speech modulates linguistic anticipatory processes. Neuropsychologia, 85, 245-255.

Rönnberg, J., Lunner, T., Zekveld, A., Sörqvist, P., Danielsson, H., Lyxell, B., et al. (2013). The ease of language understanding (ELU) model: theoretical, empirical, and clinical advances. Frontiers in Systems Neuroscience, 7:31.

Schacter, D. L., \& Wiseman, A. L. (2006). Reducing memory errors: The distinctiveness heuristic. In R. R. Hunt \& J. B. Worthen (Eds.), Distinctiveness and memory (pp. 89107). New York, NY, US: Oxford University Press.

Stadler, M. A., Roediger, H. L. III, \& McDermott, K. B. (1999). Norms for word lists that create false memories. Memory \& Cognition, 27(3), 494-500.

Stanislaw, H., \& Todorov, N. (1999). Calculation of signal detection theory measures. Behavior Research Methods, Instruments, \& Computers, 31, 137-149.

Sumner, M., \& Kataoka, R. (2013). Effects of phonetically-cued talker variation on semantic encoding. The Journal of the Acoustic Society of America, 134, EL485. 
Thapar, A., \& McDermott, K. B. (2001). False recall and false recognition induced by presentation of associated words: Effects of retention interval and level of processing. Memory \& Cognition, 29(3), 424-432.

Thomas, A. K., \& Sommers, M. S. (2005). Attention to item-specific processing eliminates age effects in false memories. Journal of Memory and Language,52(1), 71-86.

Van Engen, K. J., \& Peelle, J. E. (2014). Listening effort and accented speech. Frontiers in Human Neuroscience, 8:577.

Weil, S. A. (2003). The Impact of Perceptual Dissimilarity on the Perception of Foreign Accented Speech. Unpublished dissertation. The Ohio State University.

Witteman, M. J., Bardhan, N. P., Weber, A., \& McQueen, J. M. (2015). Automaticity and stability of adaptation to a foreign-accented speaker. Language and speech, 52(2), 168189.

Witteman, M. J., Weber, A., \& McQueen, J. M. (2013). Foreign accent strength and listener familiarity with an accent codetermine speed of perceptual adaptation. Attention, Perception, \& Psychophysics, 75(3), 537-556. 
Table 1. Summary of results of Experiment 1.

\begin{tabular}{|c|c|c|c|c|c|c|c|c|c|c|c|c|c|c|}
\hline & \multicolumn{2}{|c|}{ Recognition test } & \multicolumn{8}{|c|}{ Signal detection theory } & \multicolumn{4}{|c|}{ Recall task } \\
\hline & \multirow{2}{*}{$\frac{\text { Studied items }}{\%}$} & \multirow{2}{*}{$\frac{\text { Critical lures }}{\%}$} & \multicolumn{2}{|c|}{ A' Critical lure } & \multicolumn{2}{|c|}{$\underline{\mathrm{A}^{\prime} \text { New items }}$} & \multicolumn{2}{|c|}{ B"'dCritical lure } & \multicolumn{2}{|c|}{ B"' dew items } & \multicolumn{2}{|c|}{$\underline{\text { Studied items }}$} & \multicolumn{2}{|c|}{ Critical lures } \\
\hline & & & $\mathrm{M}$ & SD & $\mathrm{M}$ & SD & M & SD & $\mathrm{M}$ & SD & $\mathrm{M}$ & SD & $\mathrm{M}$ & SD \\
\hline Native & 82 & 89 & 0.39 & 0.18 & 0.89 & 0.05 & -0.92 & 0.11 & 0.09 & 0.59 & 8.04 & 1.09 & 0.31 & 0.27 \\
\hline Foreign & 79 & 82 & 0.41 & 0.19 & 0.88 & 0.07 & -0.86 & 0.17 & 0.19 & 0.53 & 8.09 & 1.59 & 0.30 & 0.32 \\
\hline
\end{tabular}

$\%$ = percentage of “old” responses; $\mathrm{M}$ = mean; $\mathrm{SD}$ = standard deviation. 
Table 2. Summary of results of Experiment 2.

\begin{tabular}{|c|c|c|c|c|c|c|c|c|c|c|c|c|c|c|}
\hline & \multicolumn{2}{|c|}{ Recognition test } & \multicolumn{8}{|c|}{ Signal detection theory } & \multicolumn{4}{|c|}{ Recall task } \\
\hline & \multirow{2}{*}{$\frac{\text { Studied items }}{\%}$} & \multirow{2}{*}{$\frac{\text { Critical lures }}{\%}$} & \multicolumn{2}{|c|}{ A' Critical lure } & \multicolumn{2}{|c|}{$\underline{\mathrm{A}^{\prime} \text { New items }}$} & \multicolumn{2}{|c|}{ B"'dCritical lure } & \multicolumn{2}{|c|}{ B"' dew items } & \multicolumn{2}{|c|}{$\underline{\text { Studied items }}$} & \multicolumn{2}{|c|}{ Critical lures } \\
\hline & & & $\mathrm{M}$ & SD & $\mathrm{M}$ & SD & $\mathrm{M}$ & SD & $\mathrm{M}$ & SD & $\mathrm{M}$ & SD & $\mathrm{M}$ & SD \\
\hline Native & 70 & 86 & 0.32 & 0.15 & 0.87 & 0.06 & -0.78 & 0.32 & 0.44 & 0.63 & 7.62 & 1.11 & 0.45 & 0.29 \\
\hline Foreign & 65 & 76 & 0.37 & 0.20 & 0.85 & 0.07 & -0.61 & 0.48 & 0.54 & 0.47 & 6.91 & 1.47 & 0.43 & 0.28 \\
\hline
\end{tabular}

$\%$ = percentage of “old” responses; $\mathrm{M}=$ mean; $\mathrm{SD}$ = standard deviation. 
Figure captions.

\section{False recognition of Critical lures}
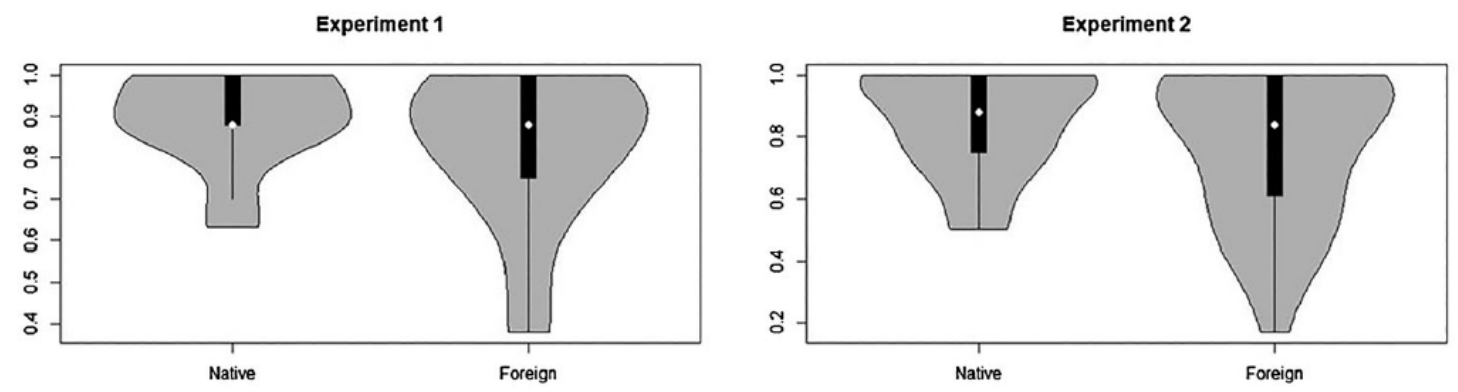

Figure 1. Violin plots showing the proportion of "old" responses and their probability density in Experiments 1 and 2, for critical lures associated to word lists spoken by native or foreign-accented speakers. The white dots represent the median values, the thick black bars show the interquartile ranges, and the thin black lines point out the lower (min) adjacent values in the data. 